\title{
RESEARCH
}

Open Access

\section{Diagnostic yield and clinical utility of a comprehensive gene panel for hereditary tumor syndromes}

\author{
Jonas Henn ${ }^{1 \dagger}$, Isabel Spier ${ }^{1,2^{*}+} \mathbb{D}$, Ronja S. Adam ${ }^{1,3}$, Stefanie Holzapfel ${ }^{2,4}$, Siegfried Uhlhaas ${ }^{1}$, Katrin Kayser $^{1}$,
} Guido Plotz ${ }^{5}$, Sophia Peters ${ }^{1}$ and Stefan Aretz ${ }^{1,2}$

\begin{abstract}
Background: In a considerable number of patients with a suspected hereditary tumor syndrome (HTS), no underlying germline mutation is detected in the most likely affected genes. The present study aimed to establish and validate a large gene panel for HTS, and determine its diagnostic yield and clinical utility.

Methods: The study cohort comprised 173 patients with suspected, but unexplained, HTS (group U) and 64 HTS patients with a broad spectrum of known germline mutations (group K). All patients in group U presented with early age at onset, multiple tumors, and/or a familial clustering of various tumor types; no germline mutation had been identified during routine diagnostics. Sequencing of leukocyte DNA was performed for the 94 HTS genes of

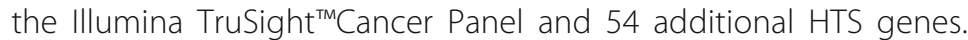

Results: The sensitivity of the panel to identify known germline variants was $99.6 \%$. In addition to known mutations, a total of 192 rare, potentially pathogenic germline variants in 86 genes were identified. Neither the proportion of rare variants per patient (group K: 0.9 variants; group U: 0.8 variants) nor the proportion of variants in the most frequently mutated, moderately penetrant genes CHEK2 and ATM showed significant inter-group difference. Four of the five patients from group $U$ with a truncating CHEK2 mutation had thyroid cancer, pointing to a broader tumor spectrum in patients with pathogenic CHEK2 variants. In $22 \%$ of patients from group K, a further potential causative variant was identified. Here, the most interesting finding was an NF1 nonsense mutation in a child with a known TP53 frameshift mutation. In 17\% of patients from group $U$, potential causative variants were identified. In three of these patients (2\%), mutations in PMS2, PTEN, or POLD1 were considered to be causative. In both groups, incidental findings with presumptive predictive value were generated.

Conclusions: The gene panel identified the genetic cause in some prescreened, unexplained HTS patients and generated incidental findings. Some patients harbored predicted pathogenic mutations in more than one established HTS gene, rendering interpretation of the respective alterations challenging. Established moderate risk genes showed an almost equal distribution among patients with known and unexplained disease.
\end{abstract}

Keywords: Familial tumor syndromes, Hereditary cancer, Tumor predisposition syndromes, Targeted sequencing, Next generation sequencing

\footnotetext{
* Correspondence: isabel.spier@uni-bonn.de

${ }^{\dagger}$ Jonas Henn and Isabel Spier contributed equally to this work.

${ }^{1}$ Institute of Human Genetics, University of Bonn, Sigmund-Freud-Str. 25,

D-53127 Bonn, Germany

${ }^{2}$ Center for Hereditary Tumor Syndromes, University of Bonn, Bonn, Germany

Full list of author information is available at the end of the article
}

(c) The Author(s). 2019 Open Access This article is distributed under the terms of the Creative Commons Attribution 4.0 International License (http://creativecommons.org/licenses/by/4.0/), which permits unrestricted use, distribution, and reproduction in any medium, provided you give appropriate credit to the original author(s) and the source, provide a link to the Creative Commons license, and indicate if changes were made. The Creative Commons Public Domain Dedication waiver (http://creativecommons.org/publicdomain/zero/1.0/) applies to the data made available in this article, unless otherwise stated. 


\section{Background}

Monogenic inherited predisposition accounts for approximately $5-8 \%$ of solid malignancies, with high variability being observed between cancer types $[1,2]$. A considerable fraction of monogenic hereditary tumor syndromes (HTS) are largely attributable to germline mutations in genes involved in cell cycle regulation, cellular proliferation, and DNA repair. To date, research has identified more than 100 cancer predisposing genes (CPG) [3]. Germline mutations in these genes represent the underlying cause of approximately 40 clinically distinct HTS, or contribute as moderately penetrant risk factors to a variety of benign and malignant tumors.

The precise identification and delineation of HTS is an important task of medical geneticists and other health care professionals, since mutation carriers have an increased lifetime risk for a largely syndrome-specific spectrum of malignancies. Prognosis in these patients can be decisively improved by early detection and treatment. This in turn can be achieved through established surveillance programs $[4,5]$; prophylactic surgery; and tailored therapies, such as PARP inhibitors in patients with BRCA1/2 mutations or PD-1 blockade in patients with microsatellite-instable colorectal cancer [6]. These approaches represent successful examples of personalized medicine.

Until recently, however, screening for a pathogenic germline mutation required the time-, cost- and personnel-intensive examination of individual genes by conventional Sanger DNA sequencing. By this, investigation was restricted to a few individual genes and to high risk groups, which were selected according to the fulfillment of specific clinical criteria (early age at onset, multiple tumors, familial clustering of tumors). These clinical criteria were therefore introduced in order to ensure a high specificity and optimize the cost-benefit ratio. However, this approach often results in low sensitivity.

Although early age at onset, multiple primary tumors and familial clustering of tumors are common features of HTS, in a considerable number of families, the established diagnostic criteria for a hereditary form are not fulfilled or overlooked due to the broad phenotypic overlap that exists between many tumor syndromes and variable phenotypes. This results in inadequate surveillance and treatment. For example, in a study by LaDuca [7], around $30 \%$ of patients with suspected hereditary colorectal cancer in whom a pathogenic germline mutation was identified using a multi-gene panel did not meet the corresponding diagnostic criteria. Moreover, in many patients with a suspected HTS, no underlying germline mutation is identified in the genes suggested by the respective tumor spectrum of the patient and the relatives.

The implementation of next generation sequencing (NGS) as high-throughput, massive parallel sequencing method, facilitates accurate and prompt diagnosis, and thus improved disease classification and patient care, through the simultaneous mutation screening of a set of relevant genes (gene panels or multi-gene analysis). Compared to Sanger sequencing, NGS is both time and resource effective $[8,9]$.

To evaluate the diagnostic yield and clinical utility of a more comprehensive screening approach, the present authors established and validated an extensive panel of HTS genes. The panel was used to screen 173 patients with a variety of suspected HTS without known pathogenic germline mutations, and 64 HTS patients with a broad spectrum of known pathogenic germline mutations. The sensitivity of the panel was validated using the confirmed germline mutations and additional polymorphisms or variants of uncertain significance (VUS), identified in the 64 HTS cases during routine diagnostics. The cohort of patients with a known pathogenic mutation was also included to demonstrate the impact of additional rare variants in genetically explained cases in comparison to the unexplained cases.

\section{Patients and methods}

Patients / data collection

All 237 index patients had been referred to the Institute of Human Genetics in Bonn from within Germany for molecular genetic investigation of a suspected HTS. Details of the patients and data collection are presented in the online material. 218 patients (92\%) were unrelated index patients.

The cohort of the present study comprised on the one hand 64 HTS index patients with a confirmed pathogenic germline mutation in an HTS gene (group K, for known mutation). These cases presented with a broad spectrum of 14 distinct HTS (Table 1A, Additional file 1: Table S1).

On the other hand 173 patients with a suspected, but genetically unexplained, HTS were included (group U, for previously unknown cause). All cases presented with an early age at onset, multiple primary tumors, and / or a striking familial clustering of various tumors and were grouped into six different phenotypic classes (Table 1B). However, no mutation in the most likely affected genes had been identified during routine diagnostics.

Prior to the present study and within the context of routine diagnostics, leukocyte DNA from almost all 237 patients was screened for germline mutations in the gene responsible for the suspected HTS and, if applicable, the most likely differential diagnoses. The number of genes investigated previously within the context of routine diagnostics are shown in the online methods and in Additional file 2: Figure S1.

\section{High-throughput targeted sequencing}

Genomic DNA was extracted from peripheral EDTAanticoagulated blood samples using the standard 
Table 1 Clinical characteristics of the total patient cohort $(n=237)$

\begin{tabular}{lcll}
\hline Phenotype group & $\begin{array}{l}\text { No. } \\
\text { Sex } \\
\text { (m / f) }\end{array}$ & $\begin{array}{l}\text { Mean age diagnosis } \\
\text { (range) }\end{array}$ \\
\hline (A) Patients with known pathogenic mutation (group K) \\
HNPCC / Lynch syndrome & 16 & $9 / 7$ & $43(25-75)$ \\
Polyposis & 25 & $15 / 10$ & $41(1-67)$ \\
Li-Fraumeni syndrome & 5 & $3 / 2$ & $17(0-44)$ \\
Cowden syndrome & 7 & $2 / 5$ & $36(1-46)$ \\
Hereditary diffuse gastric cancer & 5 & $1 / 4$ & $42(22-58)$ \\
Others & 6 & $2 / 4$ & $35(3-70)$ \\
All & 64 & $32 / 32$ & $39(0-75)$ \\
(B) Patients with unknown cause (group U) & & \\
Familial / early onset CRC & 38 & $20 / 18$ & $40(17-78)$ \\
Polyposis & 32 & $17 / 15$ & $49(7-73)$ \\
Li-Fraumeni-like syndrome & 39 & $13 / 26$ & $33(1-77)$ \\
Cowden-like syndrome & 20 & $2 / 18$ & $44(15-77)$ \\
Familial / early onset gastric & 4 & $1 / 3$ & $36(33-45)$ \\
cancer & & & \\
Others & 40 & $17 / 23$ & $39(0-71)$ \\
All & 173 & $70 /$ & $41(0-78)$ \\
& & 103 &
\end{tabular}

$C R C=$ colorectal cancer,$f=$ female, $H N P C C=$ hereditary non-polyposis colorectal cancer; $m=$ male

salting-out procedure. NGS targeted mutation screening was performed using the TruSight ${ }^{\mathrm{tm}}$ Cancer Sequencing Panel (Illumina, San Diego). This customized commercial kit includes 94 well established genes for HTS. The panel was extended using the Illumina DesignStudio by the addition of a further 54 relevant HTS genes (Additional file 3: Table S2), based on literature search. In total, 2426 selected targets were sequenced based on 3939 probes, corresponding to a cumulative target length of $626,960 \mathrm{bp}$. Library preparation, target enrichment, and high-throughput sequencing were performed according to the manufacturer's protocol. All samples were sequenced on an Illumina MiSeq sequencer. Alignment was perfomed using the software of the Illumina MiSeq sequencer or the SeqPilot software (JSI Medical Systems), based on hg19.

\section{Variant filtering and prioritization}

Data analysis was performed using the Cartagenia BENCHlab NGS platform version 3.0.4 (Leuven, Belgium). The targeted sequencing data were filtered for high-quality variants (read depth $\geq 10$ and call quality $\geq 30$ ). Afterwards truncating (nonsense mutations, frameshift deletions/ insertions and mutations at highly conserved splice sites) rare variants (minor allele frequency $(\mathrm{MAF}) \leq 0.01$ according to allele frequencies from $d b S N P$, the 1000 Genomes database (TGP), and the Exome Variant Server (EVS)) were selected. Apparent missense variants were only included if they showed an MAF of $\leq 0.001$ and were predicted to have a deleterious or damaging effect by at least two of three or three of four in silico analysis tools (PolyPhen-2, MutationTaster, LRT, and SIFT). Furthermore, rare (MAF of $\leq 0.001$ ) synonymous / silent variants were included, if they are located in the first or last three bases of an exon. Splicing efficiencies of the normal and mutant sequences were calculated using the software Alamut, which refers to the splice prediction programs Human Splicing Finder; SpliceSiteFinder-like (SSF); MaxEntScan (MES); GeneSplicer; and NNSPLICE 0.9 from BDGP (the Berkeley Drosophila Genome Project). To exclude obvious sequencing artifacts, a detailed visual inspection of the variants was performed in a read browser (SeqPilot software, JSI Medical Systems). For the evaluation of the most interesting variants, additional databases were used, in particular the Human Gene Mutation Database (HGMD); Locus-specific mutation databases (LOVD); and the Genome Aggregation Database (gnomAD).

\section{Validation of variants by sanger sequencing}

All relevant variants were validated by Sanger sequencing of the corresponding region using standard protocols. The results were analyzed with the SeqPilot software (JSI Medical Systems). To avoid pseudogene amplification, Sanger sequencing of PMS2 was based on long-range PCR with PMS2-specific primers, as described elsewhere [10].

\section{Statistical analysis}

Proportions were compared using Fisher's exact test, based on the number of patients carrying variants. All reported $p$ values are two-tailed.

\section{Results}

A total of 237 patients were included: Group U comprised 173 patients with a suspected but unexplained HTS and group K comprised 64 patients with known germline mutations. The overall workflow of the study is shown in Fig. 1.

\section{Performance of targeted sequencing}

A coverage of $\geq 10 x, \geq 20 x$, and $\geq 50 x$ was achieved for 98.6, 97.4 and $93.0 \%$ of target bases, respectively. For the $\geq 30 x$ threshold, visual inspection of the reads using the SeqPilot software revealed the following levels of exon coverage: (i) $59 \%$ of genes, all exons covered $\geq 30 x$; (ii) $35 \%$ of genes, several exons covered partially $\leq 30 x$; and (iii) $5 \%$ of genes, one or two whole exons covered $\leq 30 \mathrm{x}$ (five times exon 1 and five times exons other than exon 1) (Additional file 3: Table S2). 


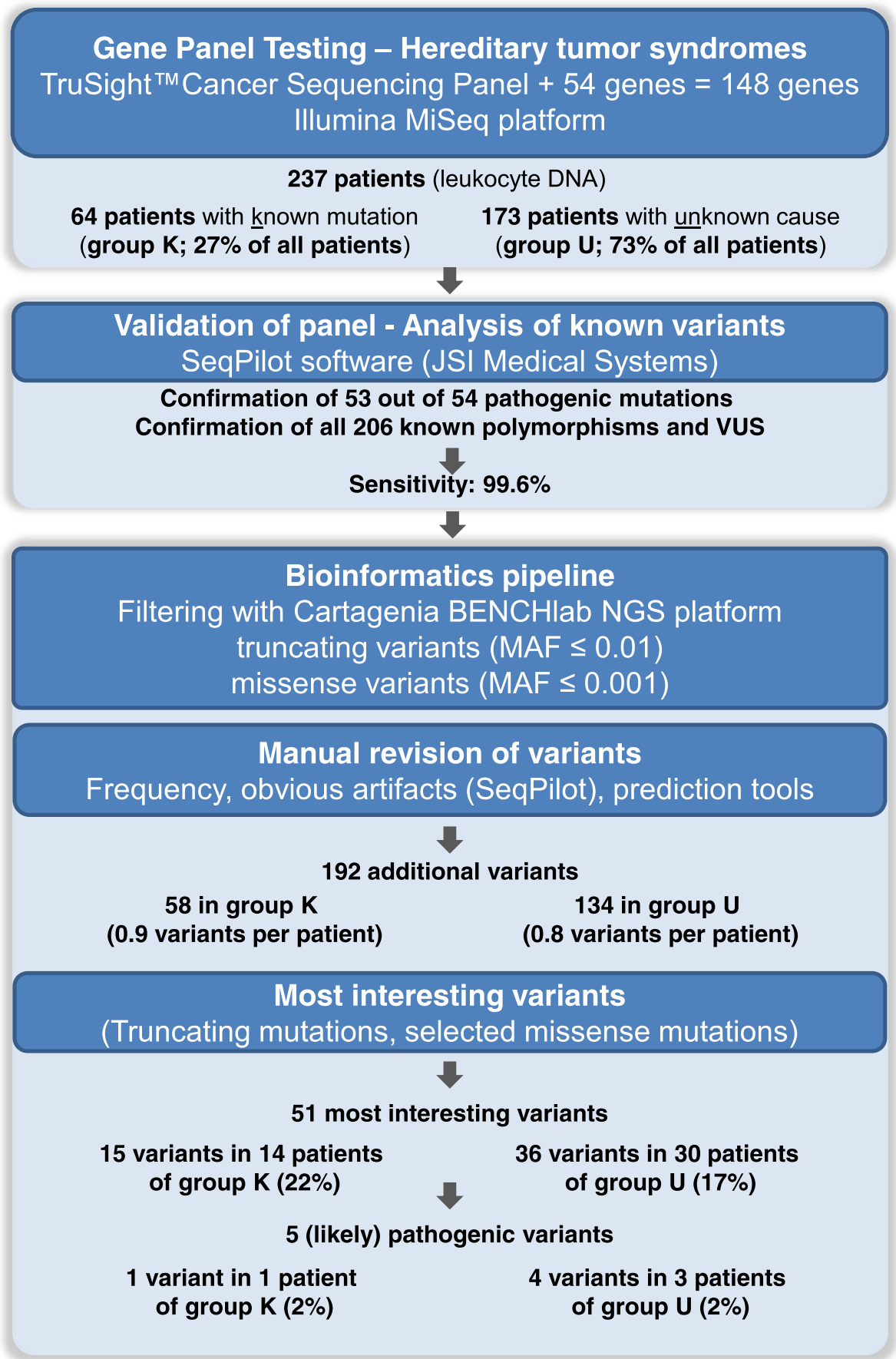

Fig. 1 Flowchart showing each step of the analysis and the number of remaining variants in patients with known pathogenic mutation (Group K) and patients with unknown cause (Group U). MAF = minor allele frequency

\section{Validation of the sensitivity of the gene panel}

To assess the analytical sensitivity of the targeted sequencing approach in terms of detecting different types of genetic variation, blind evaluation was conducted of all germline variants - including mutations, benign variants, and VUS - that had been identified by Sanger sequencing in genes examined in both patient groups during routine diagnostics. These comprised 54 known mutations and 206 benign variants or VUS. Of the known mutations, 25 represented single base pair substitutions, and 29 represented insertions and/or deletions (including six deletions/duplications with a size of 14-65 bp and two indels encompassing five to $11 \mathrm{bp}$ (Additional file 4: Table S3)). The NGS targeted sequencing panel detected 53 of the 54 
(98\%) known pathogenic germline mutations (Table 2). Two mutations were present in a homozygous state and two further mutations were present in a mosaic state (around $20 \%$ of reads). Moreover, the pipeline identified a frequent mutation in $M S H 2($ c. $942+3 \mathrm{~A}>\mathrm{T})$, which is located within a large homopolymer. A $9 \mathrm{bp}$ deletion in STK11 was not validated due to low coverage (read depth $=1 \mathrm{x}$ ). All 206 benign variants and VUS (mainly single base pair substitutions in heterozygous and homozygous state) were confirmed. Thus, the sensitivity of the gene panel to detect variants was $99.6 \%$.

\section{Diagnostic yield}

A total of 192 additional germline variants in 86 genes matched the filter criteria: 58 variants in 35 patients from group $\mathrm{K}$; and 134 variants in 92 patients from group $\mathrm{U}(p=0.8)$ (Additional file 5: Table S4, Table 3, Fig. 1). The proportion of (additional) rare variants was similar in both patient groups $(0.9$ variants per patient in group $\mathrm{K}$ and 0.8 variants per patient in group $\mathrm{U}$ ). In group $\mathrm{K}$ and $\mathrm{U}$, respectively, $45 \%$ or $47 \%$ of the patients carried no additional variant, while $47 \%$ or $47 \%$ carried 1-2 variants and $8 \%$ or $6 \%$ carried 3-4 variants (Additional file 2: Figure S2). After exclusion of the pathogenic variants (shown in bold in Table 4) a total of 187 variants must be classified currently as VUS, including 57 variants in 34 patients from group $\mathrm{K}(53 \%)$ and 130 variants in 88 patients from group U (51\%).

Of the 148 cancer panel genes, the number of additional germline variants detected was: zero in 62 genes; $1-2$ in 58 genes; and 3-12 in 28 genes (Additional file 3: Table S2, Additional file 6: Table S5, and Additional file 2: Figure S3). No significant inter-group difference was observed in the proportion of patients with variants in the most frequently mutated, moderately penetrant genes CHEK2 and ATM (group U: 18 variants in one patient each, 10\%; group K: 3 variants in 2 patients, $3 \% ; p=0.1$ ). For the other frequently mutated genes ( $\geq 3$ variants), a inter-group difference was observed for the distribution of variants. Here, variants in seven genes were detected in group $\mathrm{U}$ only $(A P C$, BMPR1A, FANCI, GALNT12, PMS2, POLD1, PTCH1); whereas variants in seven other genes were slightly overrepresented in group $\mathrm{K}(B U B 1 B, D I C E R 1, E X O 1, M S H 6$, $M T O R, P A L B 2, P O L E)$. For nearly half of the genes, the percentage of variants in genes with $\geq 3$ variants was comparable in both groups (Additional file 6: Table S5 and Additional file 2: Figure S3).

To identify further potential causative mutations among the 192 additional germline variants in both patient groups, all truncating mutations as well as start-loss and stop-loss variants were selected $(n=30)$. Of the predicted pathogenic missense variants, those were selected $(n=21)$, which were located in the most common high penetrance genes for HTS (APC, BMPR1A, BRCA1, CDH1, MLH1, MSH2, MSH6, MUTYH, NF1, PMS2, POLD1 (polymerase domain), POLE (polymerase domain), PTEN; the 192 variants did not include variants in BRCA2, SMAD4, STK11, or TP53). An overview of the 51 most interesting variants is provided in Table 4, all of them were heterozygous. All 51 variants were confirmed by Sanger sequencing (except in three cases, for whom no further DNA was available).

In both groups, the proportion of the most interesting variants per patient was 0.2 (15 variants in 14 patients of group $\mathrm{K}(22 \%)$ and 36 variants in 30 patients of group $\mathrm{U}$ $(17 \%) ; p=0.5)$ (Table 3). Interesting findings in group $\mathrm{K}$ and $\mathrm{U}$ are described below.

\section{Patients with a known causative germline mutation (group K)}

The most interesting finding was an NF1 nonsense mutation (c.4107C > A;p.Tyr1369*) in a male proband with a known TP53 frameshift mutation. He was diagnosed with a neuroblastoma and a rhabdomyosarcoma at the age of one year, and died at the age of two years. In addition, he carried a heterozygous nonsense mutation in WRN. No information was available concerning whether or not the patient exhibited cutaneous symptoms of any form of neurofibromatosis or specific symptoms of Werner syndrome.

In four patients with $A P C$-related familial adenomatous polyposis or SMAD4/BMPR1A-related juvenile polyposis syndrome, an additional potential pathogenic missense variant in an MMR gene (MLH1, MSH2, MSH6) was detected.

\section{Patients with a previously unexplained suspected HTS (group U)}

The most interesting findings included two compound heterozygous mutations in PMS2 in one patient (one start-loss mutation: c.1A > T;p.Met1? and one frameshift mutation: c.2117delA;p.Lys706Serfs*19). The phenotype

Table 2 Validation of the sensitivity of the multi-gene panel - analysis of known germline mutations, benign variants and VUS

\begin{tabular}{lllll}
\hline Group of variants & Identified by Sanger sequencing & Validated in gene panel & Sensitivity (\%) & No. of Patients \\
\hline Known pathogenic mutations & 54 & $53^{\text {a }}$ & 98 & 63 \\
Benign variants and VUS & 206 & 206 & 100 & 80 \\
All & 260 & 259 & 99.6 & 120 \\
\hline
\end{tabular}

${ }^{a}$ Non validated variant

STK11:c.907_915del9;p.lle303_Gln305del (coverage = 1)

VUS $=$ variant of uncertain significance 
Table 3 Distribution of additional rare variants and most interesting variants per patient group (Group K: Patients with known pathogenic mutation ( $n=64)$; Group U: Patients with unknown cause $(n=173)$; all patients $(n=237))$

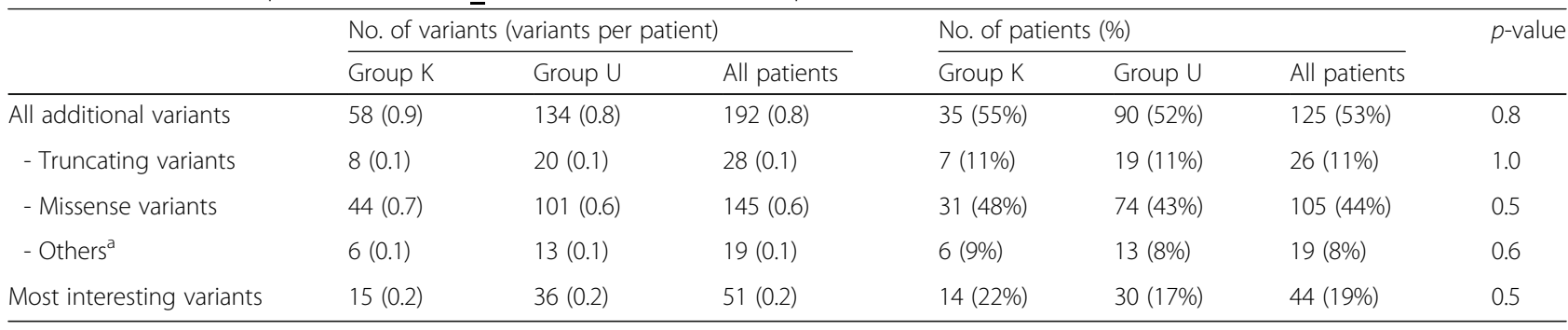

${ }^{a}$ Potential exonic splice variants; start-loss and stop-loss variants

of the patient was compatible with a constitutional mismatch repair deficiency syndrome (CMMRD) (Table 4B, ID 26356).

Furthermore, a POLD1 missense variant in the proofreading domain of the protein (c.1379 $\mathrm{T}>\mathrm{G}$;p.Leu460Arg) was identified in a patient with colorectal adenomatous polyposis and papillary renal cancer (Table 4B, ID 38569). The alteration p.Leu460Arg affects a strongly conserved leucine in the exonuclease (proofreading) domain of POLD1 (Additional file 2: Figure S4). The patient also carried a PMS2 missense variant. However, immunohistochemical analysis of the renal cancer tissue revealed no loss of PMS2 expression (or of the expression of MLH1, $\mathrm{MSH} 2$, and MSH6), and the tumor exhibited microsatellite stability.

The PTEN missense variant c.83 T > C;p.Ile28Thr was detected in one patient with early onset CRC (Table 4B, ID 40816). This variant affects a highly conserved nucleotide and amino acid in the region of a functionally important phosphatase domain of the PTEN protein. The variant has not been described previously in either patients with suspected Cowden syndrome or controls. However, pathogenic missense variants in flanking codons (e. g., codon 27 and 30) have been reported in Cowden syndrome patients [11-13].

In two more patients, possibly pathogenic variants were detected in BMPR1A and MSH6, respectively. Both were compatible with the phenotype (Table 4B, IDs 44298 and 45312).

Four of the five patients from group $\mathrm{U}$ with a truncating CHEK2 mutation had thyroid cancer (clinical details provided in Table 4B).

\section{Incidental findings in both patient groups}

Incidental (secondary) findings with presumptive predictive value were generated in both groups. This included the identification of two frameshift mutations in $S D H A$, one nonsense mutation in EXT1, one potential splice site mutation in $R A D 51 C$, and a POLD1 missense variant within the polymerase domain (c.961G > A;p.Gly321Ser). The available clinical reports contained no evidence for a personal or family history of hereditary paraganglioma-pheochromocytoma syndrome; multiple osteochondromas / exostoses; hereditary breast and ovarian cancer (HBOC); or Polymerase proofreadingassociated polyposis (PPAP).

A potential heterozygous carrier status for an autosomal recessive HTS was found in 30\% (70 / 237) of the total cohort (group K: $41 \%$ (26 / 64); group U: $25 \%$ (44 / 173)). This was based on the mode of inheritance listed in Additional file 3: Table S2.

In total, seven patients (3\%) carried two variants in the same gene (Additional file 7: Table S6). Four of these genes are known to cause recessive conditions (ERCC2 for Xeroderma pigmentosum; and MSH2, MSH6, or PMS2 for CMMRD). However, with the exception of the PMS2-related CMMRD, no patient displayed clinical signs of the respective recessive condition. In three of the four patients from group $\mathrm{K}$, the additional germline variant (appearing in the list of 192 variants) was in the same gene than the known heterozygous pathogenic germline mutation (MSH2 and MSH6, respectively), as already known using Sanger sequencing during routine diagnostics. In two of the MSH2 and MSH6 mutation carriers, segregation analysis was possible. This demonstrated that both were located on the same allele.

\section{Discussion}

In a proportion of suspected HTS patients, no germline mutation is identified in the most likely affected genes. NGS-based multi-gene analysis is a powerful approach to the detection of mutations in genes that are not primarily suspected on the basis of clinical criteria, or in HTS with several causative genes.

To determine the diagnostic yield and clinical utility of a comprehensive gene panel of 148 HTS genes, targeted sequencing was performed in 173 patients with suspected but unexplained HTS (group U) and 64 HTS patients with known pathogenic germline mutations in established HTS genes (group K).

All but one of the previously known germline mutations, benign variants, or VUS $(n=260)$ were identified, indicating a sensitivity of $99.6 \%$. This demonstrates that the multigene panel approach identified a broad spectrum of 


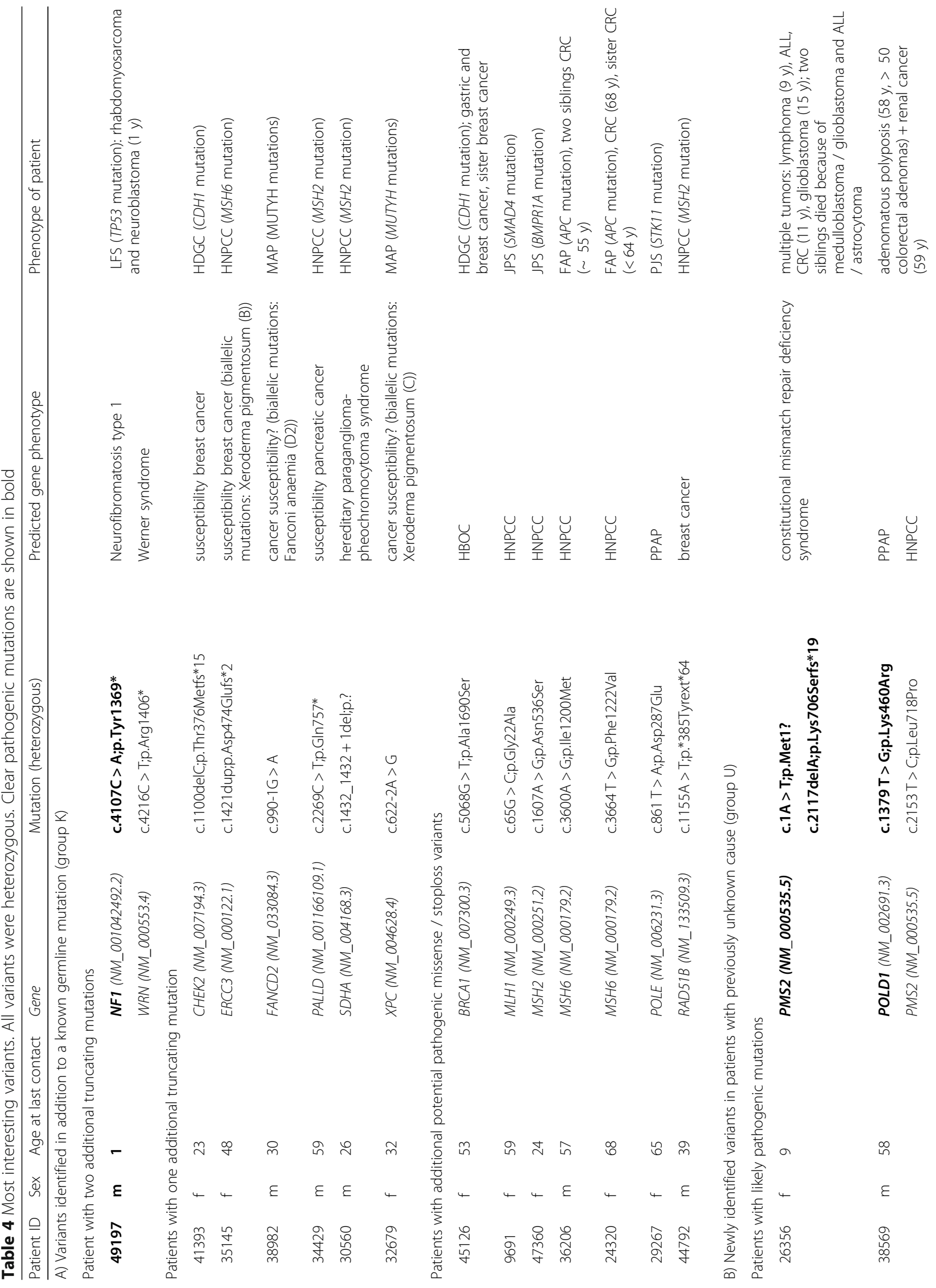




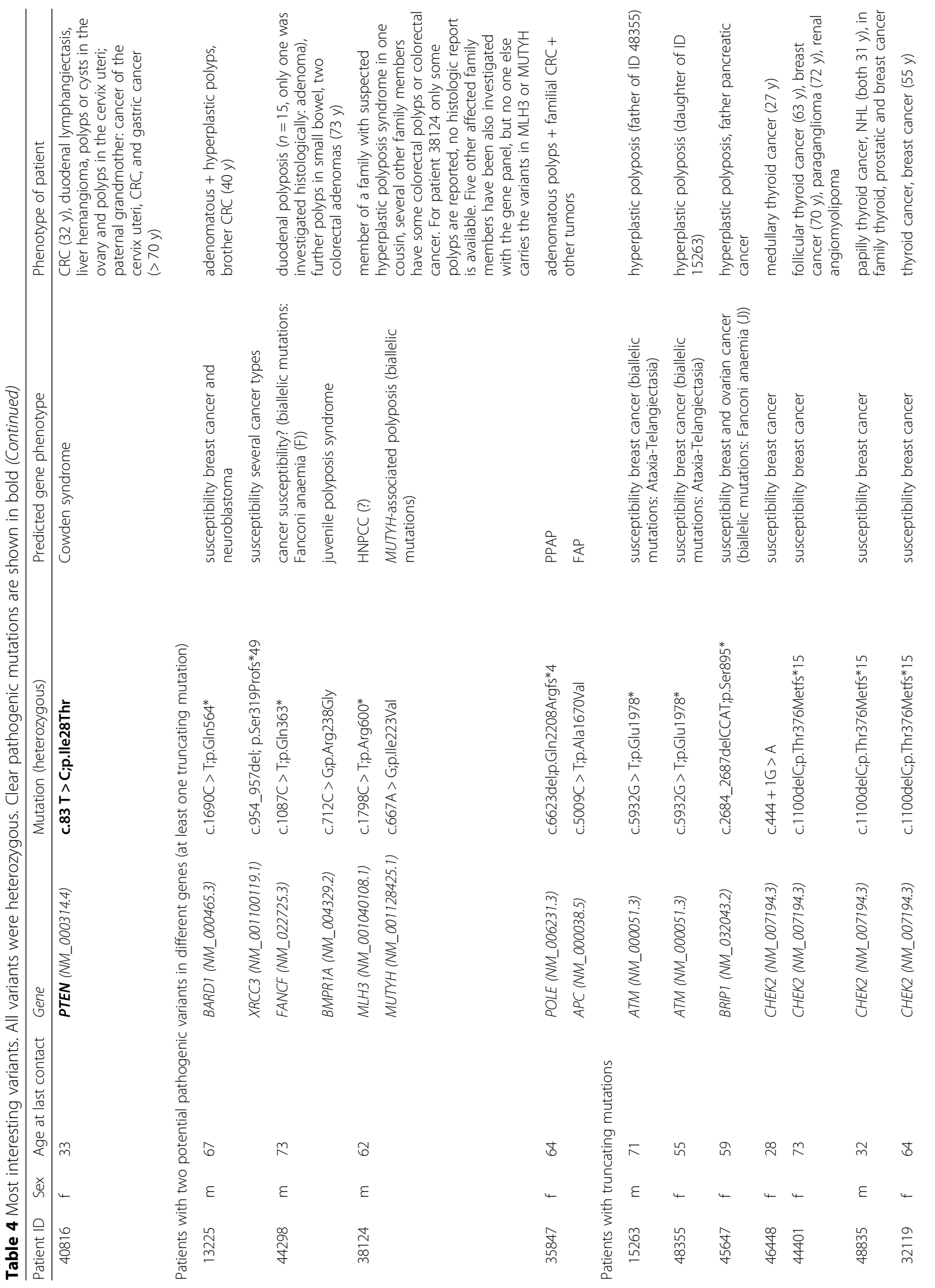




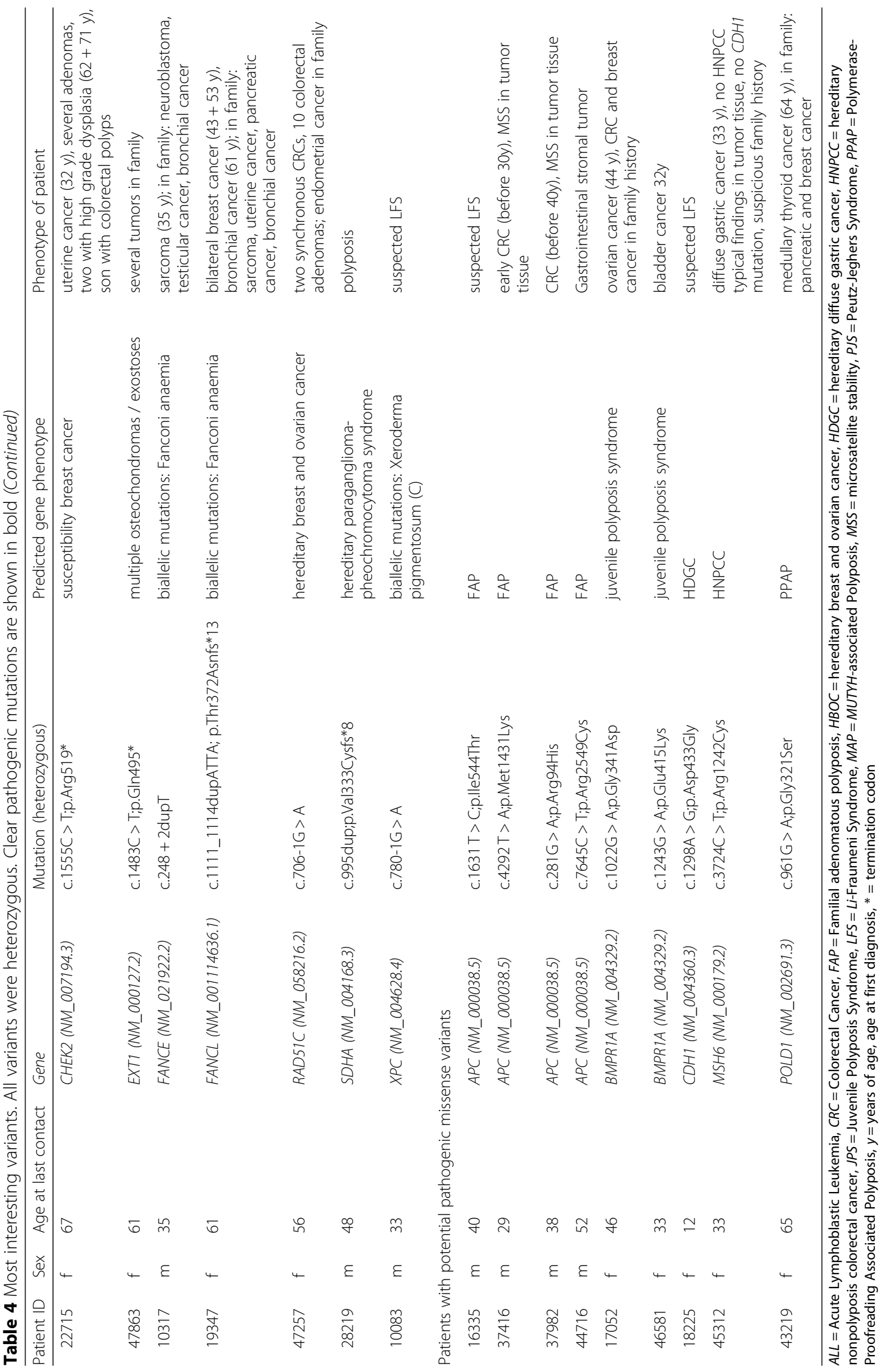


variants, ranging from single base pair substitutions to larger deletions/duplications (14-65 bp). In genes screened prior to the study during routine diagnostics by Sanger sequencing, no additional germline variant was identified by NGS.

After stringent filtering, 192 rare loss-of-function mutations and potential pathogenic missense variants remained. Interestingly, the percentage of additional germline variants was similar in both study groups. In addition, no significant inter-group difference was observed for variants in the most frequently mutated genes CHEK2 and ATM $(p=0.1)$. The identification of these variants in patients with genetically explained disease (group K) highlights the fact that caution must be exercised when interpreting their role in patients with unexplained disease, since they cannot explain the respective disease alone [14]. Moreover, the effect size of moderately penetrant risk alleles is strongly dependent on individual and family history.

Furthermore, no significant inter-group difference was found for mutation type or the presence of the 51 most interesting variants (Table 3 ). In group $\mathrm{K}$ and group $\mathrm{U}$ respectively, 22 and $17 \%$ of probands, harbored (additional) rare potential causative variants (corresponding to the most interesting variants in Table 4).

In one patient from group $\mathrm{K}(2 \%)$, a second clearly pathogenic, high penetrant germline mutation in NF1 was identified in addition to a known frameshift mutation in TP53. Due to the combination of tumors in this individual (neuroblastoma and rhabdomyosarcoma), Li-Fraumeni syndrome was suspected. However, both tumors also occur in children with neurofibromatosis type 1 [15]. A combination of pathogenic mutations would be overlooked in a stepwise approach limited to the most likely affected genes. In such patients, an atypical clinical presentation or an unusually broad spectrum of tumors might prompt the analysis of further genes. However, in routine practice, clinical information is often limited, or the patient is too young to have yet presented with the typical spectrum of signs and symptoms, thus leading to delayed diagnosis.

The present analyses also identified five patients in group U (3\%) with two potential pathogenic variants in different genes. This is consistent with the results of previous studies, which have reported rates of between 0.1 and $3 \%[7,16-18]$ or up to $7.5 \%$, when potentially pathogenic missense mutations were included [19]. These cases may involve digenic inheritance, co-inheritance of genetic modifiers, or clear pathogenic mutations [20, 21]. The presence of 2 or more inherited cancer predisposition alleles in the same individual was also described by Whitworth et al. [22] and was called multilocus inherited neoplasia alleles syndrome (MINAS).

In three patients (2\%) from group $U$, the genetic cause was identified to a high degree of certainty. The phenotype of the patient with a compound-heterozygous PMS2 germline mutation (the patient was previously described in Adam et al. [23]), was suggestive of CMMRD at the age of 15 years, however, on initial referral at the age of 9 years, the most likely differential diagnosis had been Li-Fraumeni syndrome. If multi-gene analysis had been available at that time, a CMMRD diagnosis could have been assigned much earlier and specific surveillance measures and predictive testing offered.

In a patient who showed features compatible with Cowden syndrome, a probable pathogenic PTEN missense variant was identified. Lynch syndrome had been considered the most likely clinical diagnosis at first due to the presentation of an early onset CRC and the absence of the major cancer types of Cowden syndrome, again pointing to the broad phenotypic overlap between well known tumor syndromes.

The POLD1 mutation c.1379 T > G;p.Leu460Arg in a patient with colorectal adenomatous polyposis and renal cancer is located in the vicinity of the known hotspot mutation c.1433G > A;p.Ser478Asn. Specific germline mutations in the proofreading domain of POLD1 have recently been identified as underlying rare cause of multiple colorectal adenomas and carcinomas [24]. This condition is termed Polymerase proofreading-associated polyposis (PPAP) [25]. The identified missense variant is likely to be pathogenic, since it introduces a charged side chain into the hydrophobic pocket within the exonuclease domain and thereby distorts the proteins' structure (Additional file 2: Figure S4). Very rarely affected causative genes for adenomatous polyposis such as POLD1, POLE, NTHL1, or MSH3, might collectively clarify a sufficient number of cases. However, since these polyposis types do not present with a specific phenotype, a genetic diagnosis is usually only possible using a multi-gene panel approach.

Interestingly, in the present study, rare $A P C$ missense variants were detected in group $\mathrm{U}$ only (Additional file 6: Table S5). These clustered in patients with familial / early onset CRC $(3 / 38=8 \%$ compared to the remaining patients of group $U(2 / 135=1.5 \%))$, however, the difference was not statistically significant $(p=0.07)$. Two of the variants in the present CRC patients were located in the $\beta$-catenin down-regulating domain (c.4292 T > A;p.Met1431Lys and c.5009C > T;p.Ala1670Val). Rare germline APC missense mutations are not part of the typical mutation spectrum of $A P C$-related familial adenomatous polyposis (FAP). However, research suggests that they are significantly overrepresented in patients with colorectal adenomas, particularly variants in the functionally important $\beta$-catenin down-regulating domain [26]. Notably, in two other gene panel studies, an APC missense variant was detected in $4 \%(44 / 1137)$ of patients with suspected, but unexplained, Lynch syndrome and in 5\% (53/1058) of 
unselected CRC patients [27, 28]. However, even when the results of all three studies are taken together, $A P C$ missense variants are not enriched in CRC cases (4.5\%) compared to individuals from the general population (8.9\%) (based on the gnomAD-database where 12,267 alleles with rare $(\mathrm{MAF} \leq 1 \%) A P C$ missense variants were detected in exome and genome data of 138,632 individuals). Nonetheless, the potential relevance of specific $A P C$ missense mutations cannot be excluded.

Four of the five patients from group $U$ with a truncating CHEK2 mutation had thyroid cancer (in group $\mathrm{K}$ only one patient carried a truncating CHEK2 mutation, no thyroid cancer is reported). Interestingly, a previous study with 468 unselected patients with papillary thyroid cancer found that carriers of truncating CHEK2 mutations had a six-fold increase in risk for a papillary thyroid carcinoma [29]. Thus, the tumor spectrum in patients with pathogenic CHEK2 variants seems to include thyroid cancer and appears to be broader than previously thought. However, additional larger studies are warranted to evaluate tumor risk in these patients.

Since most of the other patients with one of the most interesting variants did not show the classical phenotype, these variants might represent moderately penetrant risk alleles, or modifiers which act in conjunction with other risk factors, rather than highly penetrant mutations.

Furthermore, these variants might represent incidental (secondary) findings with presumptive predictive value. Comprehensive genetic approaches such as exome studies are prone to generate predictive information. Since the present gene panel included a limited number of causal genes for a circumscribed phenotype (HTS), the overall likelihood of predictive information was expected to be low. However, the differentiation between diagnostic and predictive information might be problematic in some cases. A predictive result was assumed if a pathogenic mutation was found and the known clinical spectrum of the altered gene did not overlap with the respective phenotype. Nonetheless, the possibility that predictive results point to a broader tumor spectrum cannot be excluded. In line with this, predictive genetic information was generated for both study groups, e. g., the identification of truncating mutations in SDHA and EXT1. Disclosure of predictive information may be challenging, and thus preand posttest counseling may be required, in particular since penetrance in this context may be reduced and thus the appropriate surveillance strategy is unclear. Particular caution is required in such cases with regard to prophylactic surgery [30]. To reduce the number of secondary findings, a panel approach (virtual panel) can be used to restrict the analyses to genes that are compatible with the phenotype of interest.

Based on the applied variant screening and filter approach, some potentially causative rare variants might have been missed in this study. In particular, large genomic rearrangements, and synonymous variants except for the first or last three exonic bases, were not considered. Since those variants might explain some more cases, the diagnostic yield might be even higher, if all possibly causative variants are included. However, the used filter and analysis approach is very similar to the current procedure in routine genetic panel diagnostics.

Previous studies have investigated the diagnostic yield of gene panels for several HTS, in particular HBOC and hereditary CRC (an (incomplete) overview of these studies is provided in Additional file 8: Table S7). The results of gene panel studies are heavily dependent on the study inclusion criteria; the number of patients (varying between 20 and 252,223); the number of previously screened genes (mostly BRCA1/2 or investigation of MMR); the number of included genes (range of 7-112); and the classification of variants. The results of these studies are therefore difficult to compare. Overall, in these studies pathogenic mutations were identified in $2-33 \%$ of patients with suspected HTS and in $2-18 \%$ of unaffected controls $[18,19]$. However, in around half of these studies, only index patients without previous Sanger sequencing of the most relevant genes were investigated, resulting in a relatively high proportion of solved cases. In virtually all previous studies, no adequate cohorts of patients with a known pathogenic mutation were included to demonstrate the impact of additional rare variants in genetically explained cases. One exception was the study of Kurian et al. [31], which investigated 57 women with known BRCA1/2 mutations. However, only one patient (2\%) carried a monoallelic MUTYH mutation.

\section{Conclusions}

In conclusion, the present data highlight the importance of analyzing all genes compatible with the phenotype of interest. The study demonstrated that the application of a comprehensive gene panel is an efficient approach to the identification of causative mutations, particularly in patients with atypical or mild phenotypes or very heterogeneous monogenic conditions. However, the diagnostic yield is limited in patients in whom a prior investigation of likely HTS genes has been conducted. The major strength of the present study was the comparison of genetically unexplained patients with a substantial group of patients with previously confirmed HTS. Moderately penetrant risk alleles occur in both patient groups and are likely to act as modifiers. The present findings also show that some patients harbor (likely) pathogenic mutations in more than one established HTS gene. This renders the interpretation of the phenotypic contribution of those alterations challenging, and indicates that the classification of a variant as pathogenic must be performed with caution. Furthermore, the potential of gene 
panels for the generation of incidental predictive information must be considered. In addition to the facilitation of diagnostics, extended analysis via comprehensive gene panels will broaden knowledge concerning the tumor spectra of established HTS genes.

\section{DATABASES / URLS}

Cancer Gene Census, Sanger Institute: http://cancer.sanger. ac.uk/cosmic/census/.

dbSNP (The Single Nucleotide Polymorphism database): www.ncbi.nlm.nih.gov/SNP/.

Ensembl Genome Browser: https://www.ensembl.org

EVS (Exome Variant Server): http://evs.gs.washington. edu/EVS/.

Genome Aggregation Database (gnomAD): http://gnomad. broadinstitute.org

HGMD (Human Gene Mutation Database): http://hgmd.org IGV (Integrative Genomics Viewer): www.broadinstitute. org/igv/.

Locus-specific mutation databases: www.lovd.nl/.

MutationTaster: www.mutationtaster.org

NCBI: www.ncbi.nlm.nih.gov/.

NNSPLICE 0.9: http://www.fruitfly.org/seq_tools/splice.html PolyPhen-2: http://genetics.bwh.harvard.edu/pph2/.

Primer3: http://bioinfo.ut.ee/primer3/

SIFT (Sorting Intolerant From Tolerant): http://sift.jcvi.org. TGP (1000 Genomes Project): www.1000genomes.org UCSC Genome Browser: http://genome.ucsc.edu

\section{Additional files}

Additional file 1: Table S1. List of all 64 patients with known germline mutations = group $\mathrm{K}$. With the exception of mutations in ${ }^{-}$MUTYH, all mutations were in a heterozygous state. (XLSX $16 \mathrm{~kb}$ )

Additional file 2: Figure S1. Number of genes investigated previously within the context of routine diagnostics and inconspicuous tumor tissue findings in cases of suspected Lynch syndrome in patients with (blue) or without (red) a known germline mutation prior to the present gene panel investigation. Figure S2. Percentage of patients with known mutations (blue) and patients without known mutations (red) carrying 0 4 additional variants. Figure S3. Percentage of patients with variants in the most frequently mutated 28 genes ( $\geq 3$ variants per gene). Figure S4. Top: Sequence logo presentation of protein sequence conservation of POLD1, comprising Leu460 and residues of relevance to its structural role. Highly conserved, hydrophobic residues significantly contributing to the hydrophobic pocket are indicated by yellow shading (mainly Leu469, Tyr472, Leu474, and Tyr396). Bottom: Overview of the structure of POLD1 and its domain architecture (left) (DOCX $527 \mathrm{~kb})$

Additional file 3: Table S2. Overview of all 148 investigated genes, including number of identified variants in the present study and details of sequencing quality (XLSX $32 \mathrm{~kb}$ )

Additional file 4: Table S3. List of detected deletions/duplications with a size of 14-65 bp and two indels encompassing five to $11 \mathrm{bp}$ (XLSX $11 \mathrm{~kb}$ )

Additional file 5: Table S4. List of the 192 additional variants that matched the filter criteria (variants in addition to a known germline mutation (group K) (A) or newly identified variants in patients with unknown cause (group U) (B)) (XLSX $30 \mathrm{~kb}$ )

Additional file 6: Table S5. List of the 28 genes with more than 3 variants per gene (XLSX $13 \mathrm{~kb}$ )
Additional file 7: Table S6. List of patients with two variants in the same gene (based on the list of 192 variants and known mutations) (XLSX $19 \mathrm{~kb})$

Additional file 8: Table S7. Literature review. (DOCX 54 kb)

Acknowledgements

The authors thank the patients and their families for participating in the study.

\section{Funding}

No funding was used for this study.

\section{Availability of data and materials}

The most relevant data generated or analysed during this study are included in the present manuscript and its supplementary information files. Further data are available from the corresponding author on reasonable request.

\section{Authors' contributions}

Study design: SA, IS. NGS experiments: SU. NGS analysis: JH, IS, SP. Sanger sequencing: JH, SU, KK, SP. Structural analysis of POLE: GP. Patient recruitment, collection of clinical and familial data: JH, IS, RA, SH, SA. Analysis of clinical data: $J H$, IS, SA. Literature search: JH, IS, SA. Figures: JH, IS, SA, GP. Preparing manuscript: JH, IS, SA. Critical review of the paper: RA, KK, GP, SP. Supervision, Expert advice: IS, SA. All authors read and approved the final manuscript.

Ethics approval and consent to participate

The patients provided informed consent to genetic diagnostics to identify the cause of the suspected HTS.

Consent for publication

Not applicable.

\section{Competing interests}

The authors declare that they have no competing interests.

\section{Publisher's Note}

Springer Nature remains neutral with regard to jurisdictional claims in published maps and institutional affiliations.

\section{Author details}

'Institute of Human Genetics, University of Bonn, Sigmund-Freud-Str. 25, D-53127 Bonn, Germany. ${ }^{2}$ Center for Hereditary Tumor Syndromes, University of Bonn, Bonn, Germany. ${ }^{3}$ Center for Experimental and Molecular Medicine, Cancer Center Amsterdam and Amsterdam Gastroenterology \& Metabolism, Amsterdam UMC, University of Amsterdam, Amsterdam, The Netherlands. ${ }^{4}$ Department of Internal Medicine I, University of Bonn, Bonn, Germany. ${ }^{5}$ Department of Medicine I, Biomedical Research Laboratory, University of Frankfurt, Frankfurt, Germany.

Received: 7 September 2018 Accepted: 12 December 2018 Published online: 23 January 2019

\section{References}

1. Lu C, Xie M, Wendl MC, Wang J, McLellan MD, Leiserson MD, et al. Patterns and functional implications of rare germline variants across 12 cancer types. Nat Commun. 2015;6:10086.

2. Huang KL, Mashl RJ, Wu Y, Ritter DI, Wang J, Oh C, et al. Pathogenic Germline Variants in 10,389 Adult Cancers. Cell. 2018;173(2):355-70.e14.

3. Rahman N. Realizing the promise of cancer predisposition genes. Nature. 2014;505(7483):302-8,

4. Provenzale D, Gupta S, Ahnen DJ, Bray T, Cannon JA, Cooper G, et al. Genetic/familial high-risk assessment: colorectal version 1.2016, NCCN clinical practice guidelines in oncology. J Natl Compr Cancer Netw. 2016; 14(8):1010-30

5. Daly MB, Pilarski R, Berry M, Buys SS, Farmer M, Friedman S, et al. NCCN guidelines insights: genetic/familial high-risk assessment: breast and ovarian, version 2.2017. J Natl Compr Cancer Netw. 2017;15(1):9-20.

6. Goyal G, Fan T, Silberstein PT. Hereditary cancer syndromes: utilizing DNA repair deficiency as therapeutic target. Familial Cancer. 2016;15(3):359-66. 
7. LaDuca H, Stuenkel AJ, Dolinsky JS, Keiles S, Tandy S, Pesaran T, et al. Utilization of multigene panels in hereditary cancer predisposition testing: analysis of more than 2,000 patients. Genet Med. 2014;16(11):830-7.

8. Sikkema-Raddatz B, Johansson LF, de Boer EN, Almomani R, Boven LG, van den Berg MP, et al. Targeted next-generation sequencing can replace sanger sequencing in clinical diagnostics. Hum Mutat. 2013;34(7):1035-42.

9. Valencia CA, Rhodenizer D, Bhide S, Chin E, Littlejohn MR, Keong LM, et al. Assessment of target enrichment platforms using massively parallel sequencing for the mutation detection for congenital muscular dystrophy. J Mol Diagn. 2012;14(3):233-46.

10. Vaughn CP, Robles J, Swensen JJ, Miller CE, Lyon E, Mao R, et al. Clinical analysis of PMS2: mutation detection and avoidance of pseudogenes. Hum Mutat. 2010;31(5):588-93.

11. Ngeow J, Stanuch K, Mester JL, Barnholtz-Sloan JS, Eng C. Second malignant neoplasms in patients with Cowden syndrome with underlying germline PTEN mutations. J Clin Oncol. 2014;32(17):1818-24

12. Vanderver A, Tonduti D, Kahn I, Schmidt J, Medne L, Vento J, et al. Characteristic brain magnetic resonance imaging pattern in patients with macrocephaly and PTEN mutations. Am J Med Genet Part A. 2014;164a(3): 627-33.

13. Bubien V, Bonnet F, Brouste V, Hoppe S, Barouk-Simonet E, David A, et al. High cumulative risks of cancer in patients with PTEN hamartoma tumour syndrome. J Med Genet. 2013;50(4):255-63.

14. Easton DF, Pharoah PD, Antoniou AC, Tischkowitz M, Tavtigian SV, Nathanson KL, et al. Gene-panel sequencing and the prediction of breastcancer risk. N Engl J Med. 2015;372(23):2243-57.

15. Brems H, Beert E, de Ravel T, Legius E. Mechanisms in the pathogenesis of malignant tumours in neurofibromatosis type 1. Lancet Oncol. 2009;10(5): 508-15.

16. Rosenthal ET, Bernhisel R, Brown K, Kidd J, Manley S. Clinical testing with a panel of 25 genes associated with increased cancer risk results in a significant increase in clinically significant findings across a broad range of cancer histories. Cancer Genet. 2017;218:219:58-68.

17. Eoh KJ. Detection of Germline Mutations in Patients with Epithelial Ovarian Cancer Using Multi-gene Panels Beyond BRCA1/2. Cancer Res Treat. 2018; 50(3):917-25.

18. Siraj AK, Masoodi T, Bu R, Parvathareddy SK, Al-Badawi IA, Al-Sanea N, et al. Expanding the spectrum of germline variants in cancer. Hum Genet. 2017; 136(11-12):1431-44.

19. DeRycke MS, Gunawardena S, Balcom JR, Pickart AM, Waltman LA, French AJ, et al. Targeted sequencing of 36 known or putative colorectal cancer susceptibility genes. Mol Genet Genomic Med. 2017;5(5):553-69.

20. Deltas C. Digenic inheritance and genetic modifiers. Clin Genet. 2018;93(3): 429-38.

21. Morak M, Massdorf T, Sykora H, Kerscher M, Holinski-Feder E. First evidence for digenic inheritance in hereditary colorectal cancer by mutations in the base excision repair genes. Eur J Cancer. 2011;47(7):1046-55.

22. Whitworth J, Skytte AB, Sunde L, Lim DH, Arends MJ, Happerfield L, et al. Multilocus inherited neoplasia alleles syndrome: a case series and review. JAMA Oncol. 2016;2(3):373-9.

23. Adam R, Spier I, Zhao B, Kloth M, Marquez J, Hinrichsen I, et al. Exome sequencing identifies Biallelic MSH3 germline mutations as a recessive subtype of colorectal adenomatous polyposis. Am J Hum Genet. 2016;99(2): 337-51

24. Palles C, Cazier JB, Howarth KM, Domingo E, Jones AM, Broderick $P$, et al Germline mutations affecting the proofreading domains of POLE and POLD1 predispose to colorectal adenomas and carcinomas. Nat Genet. 2013:45(2):136-44

25. Briggs S, Tomlinson I. Germline and somatic polymerase epsilon and delta mutations define a new class of hypermutated colorectal and endometrial cancers. J Pathol. 2013;230(2):148-53.

26. Azzopardi D, Dallosso AR, Eliason K, Hendrickson BC, Jones N, Rawstorne E, et al. Multiple rare nonsynonymous variants in the adenomatous polyposis coli gene predispose to colorectal adenomas. Cancer Res. 2008;68(2):358-63.

27. Yurgelun MB. Next-generation strategies for hereditary colorectal Cancer risk assessment. J Clin Oncol. 2015;33(5):388-93.

28. Yurgelun MB, Kulke MH, Fuchs CS, Allen BA, Uno H, Hornick JL, et al. Cancer susceptibility gene mutations in individuals with colorectal Cancer. J Clin Oncol. 2017;35(10):1086-95.
29. Siolek M, Cybulski C, Gasior-Perczak D, Kowalik A, Kozak-Klonowska B, Kowalska A, et al. CHEK2 mutations and the risk of papillary thyroid cancer. Int J Cancer. 2015;137(3):548-52.

30. Robson M. Multigene panel testing: planning the next generation of research studies in clinical cancer genetics. J Clin Oncol. 2014;32(19):1987-9.

31. Kurian AW, Hare EE, Mills MA, Kingham KE, McPherson L, Whittemore AS, et al. Clinical evaluation of a multiple-gene sequencing panel for hereditary cancer risk assessment. J Clin Oncol. 2014;32(19):2001-9.

\section{Ready to submit your research? Choose BMC and benefit from:}

- fast, convenient online submission

- thorough peer review by experienced researchers in your field

- rapid publication on acceptance

- support for research data, including large and complex data types

- gold Open Access which fosters wider collaboration and increased citations

- maximum visibility for your research: over $100 \mathrm{M}$ website views per year

At BMC, research is always in progress.

Learn more biomedcentral.com/submissions 